\title{
Fibronectin pattern in benign hyperplasia and cancer of the prostate
}

\author{
Miroslava M. Janković* and Maja M. Kosanović \\ Institute for the Application of Nuclear Energy-INEP, University of Belgrade, Belgrade, Serbia
}

\begin{abstract}
Fibronectin (FN) is a multifunctional glycoprotein involved in cell-matrix interactions. It exhibits a complex pattern of forms differing in respect to aminoacid and oligosaccharide composition. In this study we examined glycobiochemical and functional properties of the FN in benign prostatic hyperplasia (BPH) and prostatic cancer (PCa), attempting to resolve diseaserelated differences. Two BPH sera pools and three PCa sera pools were used as the FN source. The affinity-purified molecule was characterized by SDS-PAGE, immuno- and lectin blot, lectin-affinity chromatography and adhesion assay. BPH FN existed as intact molecule, giving the main immunoreactive band at $220 \mathrm{kDa}$. In contrast, PCa FN comprised three main immunoreactive fragments of 140, 110 and $90 \mathrm{kDa}$. As for glycosylation the ratio of altogether lectin-reactive PCa FN was different from that of BPH FN manifested as a decrease of Con A- and an increase of LCA-reactive moieties. Fibroblasts adhered to both FN preparations in a concentration dependent manner, but with a significantly lower efficiency to PCa FN. The results obtained showing distinct structural characteristics of PCa FN compared to BPH FN could be important for modulation of its ligand and recognition properties expressed as gain or loss of functions or as specific markers of its origin.
\end{abstract}

Keywords: Cancer marker, molecular forms, glycosylation, biological activity

\section{Introduction}

Prostate health is of special medical importance. Prostate cancer $(\mathrm{PCa})$ is the second most common cause of death from cancers in men in western countries. Approximately 218900 new cases and 27000 deaths from this malignancy were expected in during 2007 in the US alone [1]. In the EU, PCa accounts for $11 \%$ of total cancers in men and $9 \%$ of total cancer deaths [2]. On the other hand, benign prostatic hyperplasia (BPH) is also highly prevalent in older men, i.e. after 60 years of age, $60 \%$ have histological alterations and $40 \%$ obstructive and irritative symptoms [3]. The majority of new PCa cases are diagnosed as localized disease. The tumor grows slowly and its progression to locally advanced and metastatic stages varies widely and may take years [4]. As the consequence of prostate enlargement BPH progression leads to lower urinary tract

*Corresponding author: Miroslava Janković, INEP, Banatska 31b, 11080 Zemun-Belgrade, Serbia. Tel.: +381 11199 949; Fax: +381 112682 743; E-mail: miraj@inep.co.yu. symptoms and related outcomes, necessitating prostate surgery [5]. Considerable efforts have been made to improve the diagnosis and monitoring of both BPH and $\mathrm{PCa}$, especially their differential diagnosis. Although recent investigations have yielded several candidates, no markers for BPH are accepted for use in clinical practice [6]. As for $\mathrm{PCa}$, it is accompanied with marked alterations in the expression of a number of different molecules.

They comprise both biochemical and genetic markers, some of which are already used in everyday laboratory practice for diagnostic and prognostic purposes $[7$, 8]. Examinations of the structural properties of cancer biomarkers, including those related to prostate cancer are a focus of many current investigations [7-10]. On the one hand, they seem to be a promising adjunct for improving marker potential of molecules the concentrations of which are found in overlapping ranges in benign and malignant conditions. On the other hand, gaining more insight into the molecular structure of cancer biomarkers can be important for understanding the basis of tumor growth and progression and possibilities for its control and modification. 
In this study we examined the glycobiochemical and functional properties of the fibronectin $(\mathrm{FN})$ molecule in benign prostatic hyperplasia and cancer. Fibronectin is a modular multifunctional molecule involved in processes associated with cell-matrix interactions [11-14]. It exists in two main forms: a cellular form made by different cell types and a plasma form synthesized by hepatocytes. Fibronectin is a glycoprotein consisting of two similar subunits of $220 \mathrm{kD}$, linked by two disulphide bonds. Each subunit has a mosaic structure composed of three types of individual domains, but extradomains can be also included. Thus, depending on the source, FN exhibits a distinct and complex pattern of molecular forms differing in aminoacid and oligosaccharide composition. In humans, there are potentially twenty forms of FN [11-14].

This glycoprotein is expressed highly in inflamed tissue and may be an acute phase reactant $[15,16]$. Its expression is also altered during neoplastic transformation [17-20]. Thus, elevated levels of plasma or cellular FN and its fragmentation have been documented during wound healing, arthritis, bladder cancer and gastrointestinal, head and neck cancers [21-25]. As for prostate cancer, there is a limited number of experimental data indicating intensive expression in tissue as well as changes in serum concentration in cancer patients, but without information about its glycobiochemical properties [26-28]. Since, plasma FN and its fragments have been shown to exhibit specific activity towards prostate tissue i.e. a malignant prostate cell-line, in terms of stimulation or inhibition of tumor activity, gaining more insight into its structure could be of special biomedical importance [29,30].

We isolated FN from BPH and PCa serum sources and the affinity-purified products were characterized using immuno- and lectin-based techniques to resolve any disease-related structural and functional differences. The results obtained indicated distinct microheterogeneity of FN, which may have both functional and clinical relevance in relation to prostate physiology.

\section{Materials and methods}

\subsection{Materials}

Monoclonal antibody to plasma FN, clone P1H11 was purchased from R\&D Systems (Minneapolis, USA) and monoclonal anti-cellular FN antibody 1940 (EDA specific) was from Chemicon International (Temecula, CA, USA). Bovine serum albumin (BSA), acrylamide, N, N'-bis-acrylamide, Coomassie brilliant blue (CBB) and mannose (Man) were from Sigma (St. Louis, USA). Lactose and gelatin were from ICN Biochemicals (Cleveland, Ohio, USA). Sepharose 4B and Sephadex G-75 were purchased from Pharmacia Fine Chemicals AB (Uppsala, Sweden). Affinity columns with immobilized plant lectins, Vectastain Elite ABC kit, biotinylated anti-mouse $\mathrm{IgG}$, biotinylated lectins and 3, 3'-diaminobenzidine (DAB) substrate kit for peroxidase were from Vector Laboratories (Burlingame, USA). Microtest III cell culture plates of 96 wells were from Falcon, USA. Molecular mass markers RotiMark and Roti Black silver staining kit were purchased from Carl Roth GmbH+Co. (KG, Germany). CNBractivated Sepharose was from Amersham Biosciences (Uppsala, Sweden). Radioiodine $\left({ }^{125} \mathrm{I}\right)$ was from the Institute of Isotopes Co., Ltd., Budapest, Hungary. PSA concentration was determined using IRMA-PSA, a solid phase two-site immunoradiometric assay (INEP, Serbia), while FN concentration was measured with a QuantiMatrix Human Fibronectin ELISA Kit (Chemicon International, Temecula, CA, USA). Immobilion-P, polyvinylidene fluoride (PVDF) membrane was from Millipore (Bedford, USA). All other chemicals were reagent grade.

\subsection{Serum samples}

This study was carried out, according to local ethical standards, on archive specimens of sera from BPH and PCa patients seen at the Clinical Centre, Pozarevac, Serbia and INEP-Zemun, Serbia for PSA determination as part of follow up. Diagnoses were confirmed using clinically established protocols based on PSA level, physical examination, ultrasound, histomorphometry and biopsy. Pools were formed randomly with no respect to age and patient treatment. Thus, $18 \mathrm{BPH}$ sera samples were randomly divided into two groups and two pools was prepared (pool I and pool II), each consisting of 9 individual samples. PSA concentrations ranged from $0.5-3.6 \mu \mathrm{g} / \mathrm{L}$ for pool I and $0.5-3.4 \mu \mathrm{g} / \mathrm{L}$ for pool II. Samples of PCa sera were from patients diagnosed with locally advanced and advanced cancers. The PCa sera samples were taken from patients at the time when their PSA levels began to rise (after prostate cancer had been diagnosed and suitable treatment initiated) indicating disease progression. The 21 serum samples were randomly allotted to three groups and three pools was prepared (pool III, pool IV and pool $\mathrm{V}$ ), each consisting of 7 individual samples. PSA concentrations ranged from 106-986 $\mu \mathrm{g} / \mathrm{L}, 120-180 \mu \mathrm{g} / \mathrm{L}$ 
and $130-468 \mu \mathrm{g} / \mathrm{L}$ for pools III, IV and V respectively. The pools were used immediately or stored in aliquots at $-20^{\circ} \mathrm{C}$ until processed.

\subsection{Fibronectin isolation}

Fibronectin was isolated from $\mathrm{BPH}$ and $\mathrm{PCa}$ sera by affinity chromatography on a gelatin-Sepharose 4B column according to Ruoslahti et al. [31]. Gelatin was bound to $\mathrm{CNBr}$-activated Sepharose according to the manufacturer's instructions. A serum pool was placed on the gelatin-Sepharose column $(7 \mathrm{~mL})$, equilibrated with $0.05 \mathrm{M}$ PBS, pH 7.2 at room temperature and allowed to react for 3 hours. The column was washed with equilibration buffer, $0.05 \mathrm{M} \mathrm{PBS}, \mathrm{pH} 7.2$, and subsequently with $1 \mathrm{M} \mathrm{NaCl}$ until the absorbance baseline was reached. Fibronectin was then eluted with $4 \mathrm{M}$ urea at a flow rate of $1 \mathrm{~mL} / \mathrm{min}$. Fractions $(3 \mathrm{~mL})$ were collected and the elution was monitored by measuring the optical density at $280 \mathrm{~nm}$. Fractions of maxima were pooled, extensively dialyzed against $0.05 \mathrm{M}$ PBS, pH 7.2 and concentrated by ultrafiltration.

\subsection{SDS-PAGE}

SDS-PAGE was performed on $6 \%$ separating gel and $3.75 \%$ stacking gel [32] and gel was stained using the Roti Black silver staining kit according to the manufacturers' instructions. The gel was calibrated with Roti-Mark molecular mass markers.

\subsection{Immuno- and lectin-blotting}

Proteins were transferred on to polyvinylidene fluoride (PVDF) membrane by semi-dry blotting using a Multiphor II Nova Blot Unit (Pharmacia LKB, Upsala, Sweden). The conditions were: transfer buffer: $25 \mathrm{mM}$ TRIS, $192 \mathrm{mM}$ glycine, 20\% methanol pH 8.3; $1.2 \mathrm{~mA} / \mathrm{cm}^{2}$ for 1 hour. PVDF membrane was blocked with $1 \%$ casein in $0.05 \mathrm{M}$ PBS, $\mathrm{pH} 7.2$, overnight at $4^{\circ} \mathrm{C}$.

For immunoblotting, the membrane was incubated with monoclonal anti plasma-FN Ab or monoclonal anti cellular-FN Ab (both at $0.5 \mu \mathrm{g} / \mathrm{mL}$ in $1 \%$ casein 0.05 PBS, pH 7.2) overnight at $4{ }^{\circ} \mathrm{C}$, washed three times in $0.05 \%$ Tween in $0.05 \mathrm{M}$ PBS, $\mathrm{pH} 7.2$, and then incubated with biotinylated anti-mouse IgG Ab for $30 \mathrm{~min}$ at room temperature (RT). After repeating the washing procedure, the membrane was incubated for 30 min at RT with a mixture of avidin and biotinylated horse radish peroxidase (HRPO) from Elite Vec- tastain ABC kit (prepared according to the manufacturer's instructions). The membrane was then washed again three times and incubated with substrate solution containing hydrogen peroxide solution and 3, 3'diaminobenzidine (DAB) (prepared from the DAB substrate kit, according to the manufacturer's instructions), until the bands appeared.

For lectin blot, membranes were incubated with biotinylated lectins $(10 \mu \mathrm{g} / \mathrm{mL})$ for 1 hour at RT. After washing three times in $0.05 \%$ Tween in $0.05 \mathrm{M}$ PBS, $\mathrm{pH} 7.2$, a mixture of avidin and biotinylated horse radish peroxidase (HRPO) from Elite Vectastain ABC kit (prepared according to the manufacturer's instructions) was added and the whole incubated for $30 \mathrm{~min}$ at RT. The membrane was then washed again three times and incubated with substrate solution containing hydrogen peroxide solution and DAB (prepared as described above), until the bands appeared.

The corresponding negative controls (omitting the primary antibody/binding in the presence of lectincompeting sugars) were also performed and gave no visible reactions.

\subsection{Iodination}

Isolated $\mathrm{FN}$ from $\mathrm{BPH}$ pool I (BPH FN) and $\mathrm{PCa}$ pool III (PCa FN) were labeled with ${ }^{125} \mathrm{I}$, using the chloramine-T method [33]. Labeled FN was separated from free iodine on a Sephadex G-75 column, equilibrated and eluted with $0.05 \mathrm{M}$ PBS, pH 7.2 containing $0.05 \%$ bovine serum albumin. The specific activity of the iodinated preparations was $0.1 \mathrm{mCi} / \mu \mathrm{g}$ for ${ }^{125} \mathrm{I}-\mathrm{BPH} \mathrm{FN}$ and $0.2 \mathrm{mCi} / \mu \mathrm{g}$ for ${ }^{125} \mathrm{I}-\mathrm{PCa} \mathrm{FN}$.

\subsection{Lectin-affinity chromatography}

Affinity chromatography of ${ }^{125} \mathrm{I}$-FN was performed on columns of the following immobilized plant lectins: SNA (Sambucus nigra agglutinin), RCA I (Ricinus communis agglutinin I), Con-A (lectin from Canavalia ensiformis) and LCA (Lens culinaris agglutinin). The bed volume was $5 \mathrm{~mL}$ for Con-A and RCA-I, $3 \mathrm{~mL}$ for LCA and $2 \mathrm{~mL}$ for SNA columns. ${ }^{125} \mathrm{I}-\mathrm{FN}(0.4 \mathrm{~mL}$; $\sim 1000000 \mathrm{cpm}$ ) was loaded on to each of the columns. A common chromatographic scheme was applied to all columns, according to the manufacturer's instructions. After 3 hours incubation at room temperature, the fractions $(1 \mathrm{~mL}$ for $5 \mathrm{~mL}$ columns or $0.5 \mathrm{~mL}$ for $2 \mathrm{~mL}$ and $3 \mathrm{~mL}$ columns) were collected. The unbound and the retarded fractions from SNA and RCA I columns were eluted with binding buffer (0.05 M PBS pH 7.2 
for SNA and RCA I and 0.1 M acetate buffer $\mathrm{pH}$ 6.0, supplemented with $100 \mathrm{mM} \mathrm{CaCl} 2, \mathrm{MgCl}_{2}$ and $\mathrm{MnCl}_{2}$ for Con-A and LCA columns). The bound fractions were specifically eluted by addition of competitive sugars: $0.1 \mathrm{M}$ mannose (for Con-A and LCA) and $0.1 \mathrm{M}$ lactose (for SNA and RCA). Finally, the tightly bound fraction was eluted with low $\mathrm{pH}$ buffers: $0.1 \mathrm{M}$ glycin$\mathrm{HCl} \mathrm{pH} 3.0$ (for RCA), $0.2 \mathrm{M}$ acetic acid (for LCA) or $0.3 \mathrm{M}$ lactose in $0.2 \mathrm{M}$ acetic acid (for SNA). The Con-A column was finally eluted with $0.1 \mathrm{M}$ borate buffer, pH 6.5. Elution of ${ }^{125} \mathrm{I}-\mathrm{FN}$ was monitored by measuring the radioactivity of each fraction.

\subsection{Fibroblast adhesion assay}

COS-7 fibroblasts were grown in Dulbecco's minimal essential (DME) medium supplemented with $10 \%$ heat-inactivated fetal bovine serum (FCS), $100 \mathrm{U} / \mathrm{mL}$ penicillin and $100 \mu \mathrm{g} / \mathrm{mL}$ streptomycin [34,35].

The adhesion assay was performed according to Hyytiainen and Keski-Oja [36]. Cell culture plates of 96 wells were coated with serial dilutions of a) $\mathrm{BPH}$ FN/PCa FN (isolated from BPH pool I (BPH $\mathrm{FN}$ ) and PCa pool III (PCa FN) (starting concentration $26.5 \mu \mathrm{g} / \mathrm{mL})$ or b) monoclonal anti- FN Ab $(0.1 \mu \mathrm{g} / 50$ $\mu \mathrm{L}$ per well) for 1 hour at RT.

a) FN coated wells: after washing three times with $200 \mu \mathrm{L} 0.05 \mathrm{M}$ PBS, pH 7.2, $100 \mu \mathrm{L} \mathrm{1 \%} \mathrm{BSA} \mathrm{(heat}$ treated at $70^{\circ} \mathrm{C}$ ) was added and incubated for 1 hour at RT to block non-specific binding. The washing procedure was repeated and the cell suspension was added to FN-coated wells $\left(5 \times 10^{4}\right.$ fibroblast cells in $50 \mu \mathrm{L} \mathrm{FCS}$ free DME medium) and incubated for 1 hour at $37^{\circ} \mathrm{C}$. Non attached cells were removed by gentle washing three times with $200 \mu \mathrm{L} 0.05 \mathrm{M}$ PBS, pH 7.2. Cells were then fixed with $4 \%$ paraformaldehyde for $10 \mathrm{~min}$, washed and stained with $0.1 \%$ Coomassie brilliant blue in $30 \%$ methanol and $10 \%$ acetic acid. The excess of color was removed with $30 \%$ methanol and $10 \%$ acetic acid and the cells were finally washed with $0.05 \mathrm{M}$ PBS, pH 7.2. The cells were lysed with 1\% SDS and $\mathrm{OD}$ at $620 \mathrm{~nm}$ was measured using Microplate Reader LKB5060-006 (LKB, Austria).

b) Antibody coated wells: after washing three times with $200 \mu \mathrm{L} 0.05 \mathrm{M}$ PBS, pH 7.2, $100 \mu \mathrm{L} \mathrm{1 \%} \mathrm{BSA}$ (heat treated at $70^{\circ} \mathrm{C}$ ) was added and incubated for 1 hour at RT to block non-specific binding. The washing procedure was repeated and then $\mathrm{BPH}$ and $\mathrm{PCa}$ pooled sera (FN concentration range: $0.4-162.0 \mu \mathrm{g} / \mathrm{mL}$ ) was added and incubated for 1 hour at $37^{\circ} \mathrm{C}$. After another cycle of washing the cell suspension was added ( 5 $\times 10^{4}$ fibroblast cells in $50 \mu \mathrm{L}$ FCS free DME medium) and incubated for 1 hour at $37^{\circ} \mathrm{C}$. Non attached cells were removed by gentle washing three times with $200 \mu \mathrm{L} 0.05 \mathrm{M}$ PBS, pH 7.2. Cells were then fixed with $4 \%$ paraformaldehyde for $10 \mathrm{~min}$, washed and stained with $0.1 \%$ Coomassie brilliant blue in $30 \%$ methanol and $10 \%$ acetic acid. The excess of color was removed with $30 \%$ methanol and $10 \%$ acetic acid and the cells were finally washed with $0.05 \mathrm{M}$ PBS, $\mathrm{pH} 7.2$. The cells were lysed with $1 \%$ SDS and OD at $620 \mathrm{~nm}$ was measured using the Microplate Reader LKB5060-006 (LKB, Austria).

All samples were tested in duplicate, and the results are presented as the mean value with error bars representing the standard error. Each assay was performed three times.

\section{Results}

\subsection{Molecular forms of BPH FN and PCa FN}

Representative SDS-PAGE and immunoblots of affinity purified BPH FN and PCa FN are shown in Fig. 1(A, B). BPH FN existed predominantly as an intact molecule, which, under reducing conditions, gave a main diffuse band at $220 \mathrm{kDa}$ corresponding to the molecular mass of the FN subunit (Fig. 1.A.2, BPH $\mathrm{FN})$. It was immunoreactive with monoclonal anti-FN (Fig. 1B, BPH FN).

Under non-reducing conditions the PCa FN preparation gave one higher molecular mass immunoreactive band at the interface of the stacking and separating gel, possibly corresponding to FN multimers (Fig. 1.A.2, PCa FN and Fig. 1B, PCa FN). Under reducing conditions, in contrast to BPH FN, three bands corresponding to 140,110 and $90 \mathrm{kDa}$ were dominant (Fig. 1.A.2, $\mathrm{PCa}$ FN) and their identities as FN fragments were confirmed by immunoblot analysis (Fig. 1B, PCa FN). Both preparations contained low molecular mass bands possibly corresponding to artifactual fragmentation, which may not be avoidable during the experimental procedures. In addition, none of the isolated BPH FN and $\mathrm{PCa} F \mathrm{FN}$ preparations were immunoreactive with monoclonal anti-EDA+ antibody specific for cellular FN (data not shown). The results presented are related to pools of BPH sera (pool I, $n=9$, PSA range 0.5$3.6 \mu \mathrm{g} / \mathrm{L}$ ) and PCa sera (pool III $n=7$, PSA range 106-986 $\mu \mathrm{g} / \mathrm{L})$.

Although, total binding and yield were different, comparable results were obtained for the other indepen- 
A

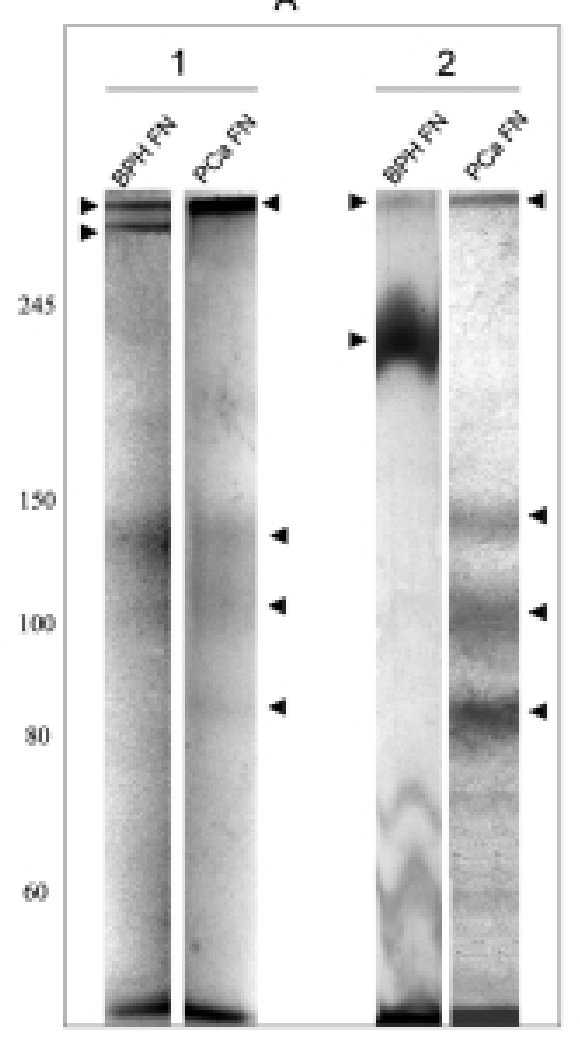

B

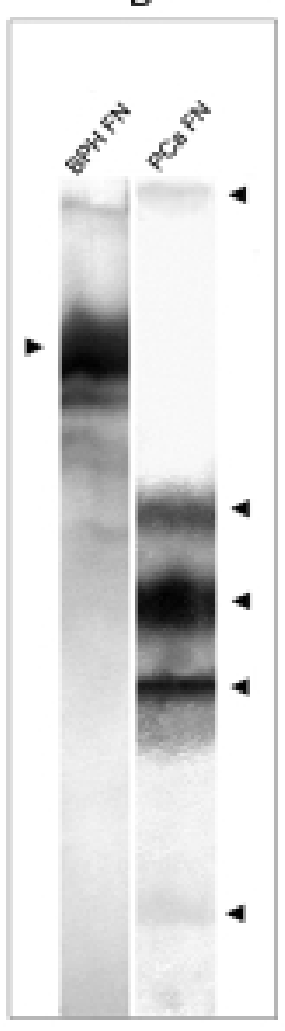

Fig. 1. SDS-PAGE and immunoblot of affinity-purified FN preparations. Fibronectin preparations, isolated from pools of BPH (pool I, $n=$ 9, PSA range 0.5-3.6 $\mu \mathrm{g} / \mathrm{L}$ ) and PCa (pool III, $n=7$, PSA range 106-986 $\mu \mathrm{g} / \mathrm{L}$ ) sera, by gelatin-affinity chromatography, were resolved on $6 \%$ separating and 3.75\% stacking gel, and silver stained (A) or immunoblotted (B). Panel A (silver staining): BPH FN and PCa FN subjected to SDS-PAGE, under non-reducing (1) and reducing (2) conditions. Panel B (immunoblotting): after SDS-PAGE, under reducing conditions, proteins were transferred onto membrane and probed with monoclonal anti-FN antibody clone P1H11. Bound antibody was visualized using a mixture of avidin and biotinylated-HRPO followed by addition of $\mathrm{H}_{2} \mathrm{O}_{2} / 3,3$ '-diaminobenzidine. Numbers indicate molecular mass (kDa).

dently examined pools of PCa sera (pool IV, $n=7$, PSA range $120-180 \mu \mathrm{g} / \mathrm{L}$, and pool $\mathrm{V}, n=7$, PSA range $130-468 \mu \mathrm{g} / \mathrm{L}$ ) and BPH (pool II, $n=9$, PSA range $0.5-3.4 \mu \mathrm{g} / \mathrm{L})$. This indicates that the different qualitative pattern of $\mathrm{FN}$-immunoreactive molecular forms was not due to sample to sample variations.

\subsection{Glycosylation of BPH FN and PCa FN}

The results of carbohydrate composition analysis of $\mathrm{BPH}$ FN and PCa FN under non-reducing and reducing condition using lectin blots are shown in Fig. 2A and Fig. 2B, 2C respectively. The following lectins were used: Concanavalin A, Con A (specific for the mannosyl core of high mannose-, hybrid- and bianntenary complex-type of N-glycans), Sambucus nigra agglutinin, SNA (specific for sialic acid $\alpha 2,6$ linked
Gal/GalNAc), Ricinus communis agglutinin I, RCA I (specific for Gal $\beta 1,4 \mathrm{GlcNAc}$ ), Lens culinaris agglutinin, LCA (specific for $\alpha 1,6$ core fucose). Generally, lectin-binding to both BPH FN and PCa FN was more noticeable when reduced samples were analyzed, probably due to exposure of lectin-reactive moieties.

Thus, the main immunoreactive BPH FN band at $220 \mathrm{kDa}$ was bound by RCA I, SNA, Con-A and weakly by LCA (Fig. 2B). These lectins reacted differently with the three immunoreactive bands of PCa FN, observed (Fig. 2C). Thus, SNA and RCA I bound to the $90 \mathrm{kDa}$ and $110 \mathrm{kDa}$ bands, which were also less strongly recognized by Con-A and LCA, respectively. As for $140 \mathrm{kDa}$ immunoreactive band, it was stained only with RCA I.

Microheterogeneity of the oligosaccharide chains of BPH FN and PCa FN was analyzed by lectin-affinity 

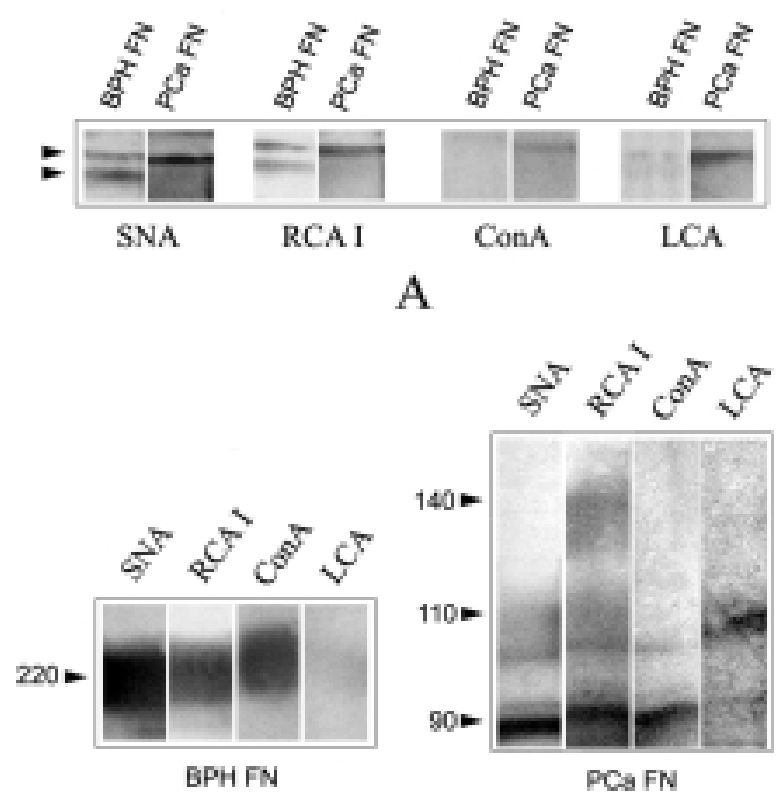

B

$\mathrm{C}$

Fig. 2. Lectin-blot analysis of affinity-purified FN preparations. Fibronectin preparations, isolated from pools of BPH (pool I) and PCa (pool III) sera, by gelatin-affinity chromatography, were resolved on $6 \%$ separating and $3.75 \%$ stacking gel, blotted on membrane and probed with biotinylated lectins: SNA (Sambucus nigra agglutinin), RCA I (Ricinus communis agglutinin I), Con-A (lectin from Canavalia ensiformis) and LCA (Lens culinaris agglutinin). Bound lectins were visualized using a mixture of avidin and biotinylated-HRPO followed by addition of $\mathrm{HO}_{2}$ and 3,3'-diaminobenzidine. Panel A: lectin blots of BPH FN and PCa FN, resolved under non-reducing conditions (see Fig. 1.A.1). Panel B: lectin blots of BPH FN, resolved under reducing conditions (see Fig. 1.A.2). Panel C: lectin blots of PCa FN, resolved under reducing conditions (see Fig. 1.A.2). Numbers indicate molecular mass (kDa).

chromatography using radiolabeled samples. The relative abundances of fractions eluted with sugar solution or low $\mathrm{pH}$ buffer, which separated high affinity bound glycoforms from weakly bound ones were, recorded (Fig. 3A, 3B). The results obtained indicated that the FN preparations comprised glycoforms differing in relative abundance and lectin-reactivities. The Con-Areactive fraction of $\mathrm{BPH}$ FN was most abundant, followed by SNA- $(46 \%)$ and RCA I- $(32 \%)$ reactive fractions. The LCA-reactive fraction $(20 \%)$ was the lowest (Fig. 3A). On the other hand, the relative amount of altogether immuno/lectin-reactive $\mathrm{PCa} F \mathrm{FN}$ was highest for RCA I- and SNA-, followed by Con-A- (72\%) and LCA- bound fraction (59\%) (Fig. 3B).

\subsection{Adhesion of fibroblasts to BPH FN and PCa FN}

COS-7 fibroblast adhesion to BPH FN and PCa FNcoated wells are shown in Fig. 4A. Cells adhered to both FN preparations in a concentration dependent manner (concentration range $0.2-26.5 \mu \mathrm{g} / \mathrm{mL}$ ), but with a significantly lower efficiency to PCa FN coated wells (Fig. 4A).

Similar results were observed when pools of BPH and $\mathrm{PCa}$ sera were used as the FN source instead of affinity-purified preparations, suggesting that the effect observed was not due to molecular modification and degradation during experimental procedures. Thus, monoclonal anti-FN Ab were adsorbed to cell-culture plates and allowed to react with pools of $\mathrm{BPH}-$ or $\mathrm{PCa}-$ sera, after which COS-7 fibroblasts were added and incubated at $37^{\circ} \mathrm{C}$ for one hour to attach to Ab-bound FN (Fig. 4B). The results obtained indicated adhesion efficiency to BPH FN increased at concentrations between $4 \mu \mathrm{g} / \mathrm{mL}$ and $20 \mu \mathrm{g} / \mathrm{mL}$, after which saturation was reached.

\section{Discussion}

The data we obtained for BPH FN and PCa FN pointed to differences in their protein and oligosaccharide 

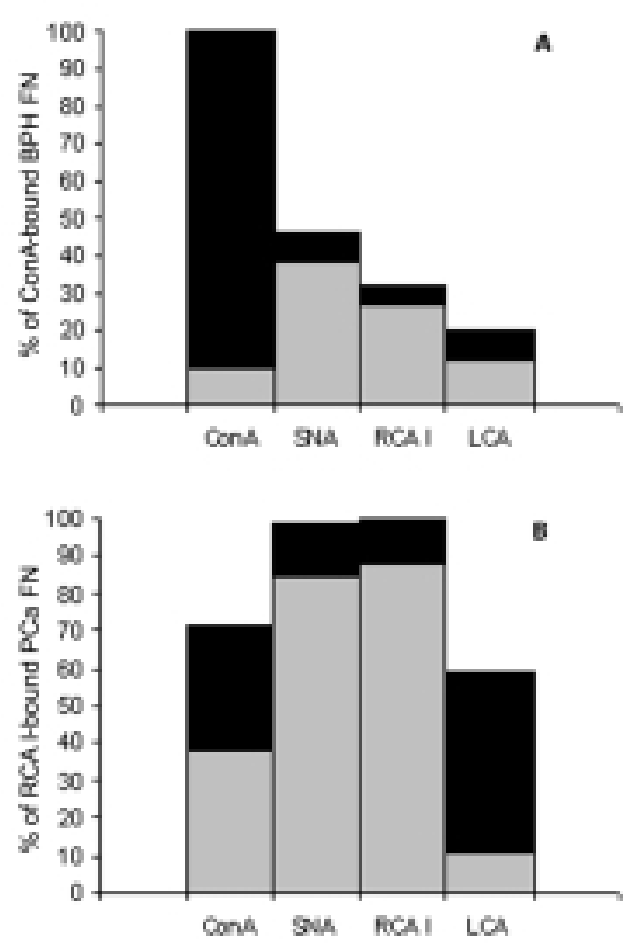

Fig. 3. Microheterogeneity of BPH FN and PCa FN: Fibronectin preparations, isolated from pools of BPH (pool I) and PCa (pool III) sera, by gelatin-affinity chromatography, were iodinated and subjected to lectin-affinity chromatography to detect the presence of different FN glycoforms. The following lectins were used as ligands: SNA (Sambucus nigra agglutinin), RCA I (Ricinus communis agglutinin I), Con-A (lectin from Canavalia ensiformis) and LCA (Lens culinaris agglutinin). A common chromatographic scheme was applied to all columns. The unbound and the retarded fractions were eluted with binding buffers. The bound fractions were specifically eluted by addition of competitive sugars or low $\mathrm{pH}$ buffers as indicated in Materials and methods. The percentage of sugar-elutable (grey) and low $\mathrm{pH}$ buffer-elutable (dark) fractions was calculated relative to the total bound radioactivity to the most reactive lectin. A) BPH FN; $y$ axis: \% of BPH FN bound to ConA as the lectin most reactive with BPH FN; B) PCa FN; y-axis: \% of PCa bound to RCA I as the lectin most reactive with $\mathrm{PCa} \mathrm{FN}$.

composition seen as distinct patterns of molecular mass forms and lectin-reactive forms.

BPH FN showed the pattern characteristic of normal plasma FN, which differed from PCa FN mainly in respect to the presence of FN fragments. Three immunoreactive fragments having molecular masses of 140, 110 and $90 \mathrm{kD}$ were dominant, the last one detected specifically in PCa. Generally, FN fragments are reported to be characteristic of various pathological conditions. They can be generated through metalloproteinase or kallikrein activity and released from tissue into the circulation $[20,21,37,38]$. BPH and PCa are accompanied with elevated serum concentrations of kallikrein-3, but no correlation with FN concentration in cancer has been found [28]. However, the available experimental data are different, indicating both decrease and increase of FN level in cancer [26-28,38, 39].

$\mathrm{FN}$ is a glycoprotein containing $5 \%$ carbohydrates linked as both N- and O-glycans [14]. Depending on the source, FN exhibits differences in degree of sialylation and the type of linkage of sialic acid, as well as in the absence or presence of fucose [40-46]. The $\mathrm{N}$-glycans of plasma FN are complex-type bianntenary oligosaccharides, largely sialylated with predominance of the NeuAc alpha 2,6Gal linkage, whereas fetal/oncofetal FN, which is upregulated during development/transformation, is core fucosylated and generally not or poorly sialylated, with predominance of the NeuAc alpha 2,3Gal linkage. In addition, neoplastic transformation is generally accompanied by greater branching and increased carbohydrate content of FN, but the available data are mainly related to cellular $\mathrm{FN}$ forms, whereas data on plasma $\mathrm{FN}$ in cancer are scarce [47-51].

The analysis of the carbohydrate composition of BPH FN was in general agreement with the results reported for normal plasma $\mathrm{FN}$ regarding sialylation and fucosylation. In comparison to $\mathrm{BPH} \mathrm{FN}$, the ratio of altogether lectin-reactive PCa FN was different and manifested as a decrease of Con-A- and an increase of LCA-reactive forms. Since, PCa FN comprised different fragments, this should be born in mind when the results of glycosylation analysis are considered. PCa FN fragments have molecular masses corresponded to the cell-binding and gelatin-binding domain-containing fragments. It is known that the gelatin-binding domain on the FN molecule is mostly glycosylated, but the cellbinding domain also contains a significant amount of carbohydrates [14].

FN exhibits various biological activities through its different domains [12,14]. It is involved in cellular migration, adhesion, morphology and spreading, cytoskeletal organization, oncogenic transformation, phagocytosis, hemostasis/thrombosis embryonic differentiation etc [12,52]. These activities are mediated through its interactions with different molecules such as gelatin, heparin, integrins, actin, DNA, fibrin, hyaluronic acid, proteoglycans/gangliosides, thrombospondin, complement factor C1q [12,52]. Some of these activities might be influenced by glycosylation [12,14]. Thus, it was shown that inhibition of FN glycosylation increases its gelatin-binding and cell- 

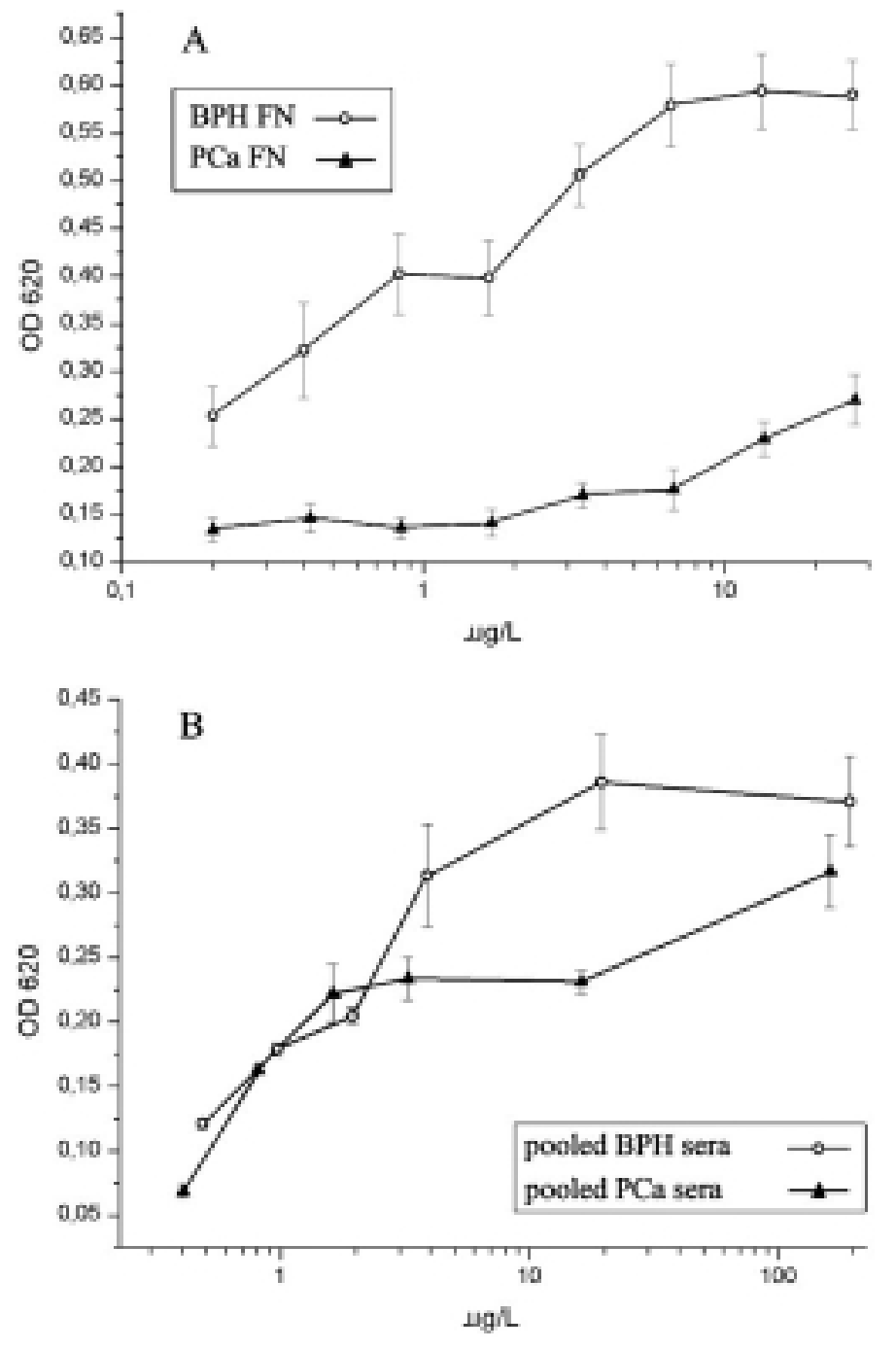

Fig. 4. COS-7 fibroblast adhesion to FN. A) Affinity purified FNs, isolated from pools of BPH (pool I) and PCa (pool III) sera, by gelatin-affinity chromatography, were adsorbed to cell-culture plates in the concentration range: $0.2-26.5 \mu \mathrm{g} / \mathrm{mL}$. COS-7 fibroblasts were added $(5 \times 1 \%$ cells/well) and incubated for 1 hour at $37^{\circ} \mathrm{C}$. After plating, cells were fixed, stained with Coomassie brilliant blue and then lysed with SDS after which the OD at $620 \mathrm{~nm}$ was measured. All samples were tested in duplicate, and the results are presented as the mean value with error bars representing the standard error.

B) Monoclonal anti-FN antibody, clone P1H11 were adsorbed to cell-culture plates $(0.1 \mu \mathrm{g} / \mathrm{well})$, and allowed to react with pools of BPH- or $\mathrm{PCa}$-sera (FN concentration range: $0.4-162.0 \mu \mathrm{g} / \mathrm{mL})$ for 1 hour at $37 \mathrm{C}$. COS-7 fibroblasts were added $\left(5 \times 10^{4}\right.$ cells/well) and incubated at $37^{\circ} \mathrm{C}$ for 1 hour to attach to antibody-bound FN. After plating, cells were fixed, stained with Coomassie brilliant blue and then lysed with SDS after which OD at $620 \mathrm{~nm}$ was measured. All samples were tested in duplicate, and the results are presented as the mean value with error bars representing the standard error.

spreading potency, but not heparin-binding, and that the presence of $\mathrm{N}$-linked polylactosamine chains decreased the affinity for gelatin [53].

Our results showed that BPH FN and PCa FN differed in potency in the fibroblast adhesion assay, both as isolated preparations or as serum samples. Recent data indicated that the major fraction of FN present in ECM of tissue is plasma derived, suggesting that it has a general and direct role in cellular processes and maintenance of tissue integrity [54,55]. Concerning the molecular aspects of prostate cancer development, plasma FN is of interest due to its potency to stimulate invasion and metastatic spread and to protect prostate cancer cells from tumor necrosis factor [29,30,56-58]. Thus, the metastatic PCa cell, unlike the normal cell, can be stimulated by intact or distinct fibronectin frag- 
ments to invade surrounding tissue.

The results obtained here showing some distinct structural characteristics of plasma FN in PCa compared to BPH could be important for modulation of its ligand and recognitive properties in terms of gaining or losing functions or as specific markers of its origin. This favors further work to explore these characteristics from both basic and clinical aspects.

\section{Acknowledgement}

The authors thank dr Snezana Kojic from IMGGE, for providing COS-7 cell line. This work was supported by the Ministry for Science of the Republic of Serbia, project code 143048 .

\section{References}

[1] A. Jemal, R. Siegel, E. Ward, T. Murray, J. Xu and M.J. Thun, Cancer statistics, 2007, CA Cancer J Clin 57(1) (2007), 43-66.

[2] R.J. Black, F. Bray, J. Ferlay and D.M. Parkin, Cancer incidence and mortality in the European Union: cancer registry data and estimates of national incidence for 1990, Eur J Cancer 33 (1997), 1075-1107.

[3] C.J. Girman, Population-based studies of the epidemiology of benign prostatic hyperplasia, Br J Urol 82 (Supl 1) (1998), 34-43.

[4] G.W. Chodak, R.A. Thisted, G.S. Gerber, J.E. Johansson, J. Adolfsson, G.W. Jones, G.D. Chisholm, B. Moskovitz, P.M Livne and J. Warner, Results of conservative management of clinically localized prostate cancer, $N$ Engl J Med 330(4) (1994), 242-248.

[5] M.J. Barry, F.J. Jr. Fowler, L. Bin, J.C. 3rd Pitts, C.J. Harris and A.G. Jr. Mulley, The natural history of patients with benign prostatic hyperplasia as diagnosed by North American urologists, J Urol 157(1) (1997), 10-14.

[6] G.W. Cannon and R.H. Getzenberg, Biomarkers for benign prostatic hyperplasia progression, Current Prostate Reports 5(3) (2007), 121-125.

[7] J.V Tricoli, M. Schoenfeldt and B.A, Detection of prostate cancer and predicting progression: current and future diagnostic markers, Clin Cancer Res 10(12Pt1) (2004), 3943-3953.

[8] H.G. van der Poel, Molecular markers in the diagnosis of prostate cancer, Crit Rev Oncol Hematol 61(2) (2007), 104139.

[9] M.M. Janković and M.M. Kosanović, Glycosylation of urinary prostate-specific antigen in benign hyperplasia and cancer: assessment by lectin-binding patterns, Clin Biochem 38(1) (2005), 58-65.

[10] S. Golubovic and M. Jankovic, Glycobiochemical characterization of salivary carcinoembryonic antigen, J BUON 12(2) (2007), 269-275.

[11] D.F. Mosher, Fibronectin, Prog Hemost Thromb 5 (1980), 111-151.

[12] R.O. Hynes and K.M. Yamada, Fibronectins: multifunctional modular glycoproteins, J Cell Biol 95(2 Pt 1) (1982), 369-377.
[13] C. Ffrench-Constant, Alternative splicing of fibronectin-many different proteins but few different functions, Exp Cell Res 221(2) (1995), 261-271.

[14] R. Pankov and K.M. Yamada, Fibronectin at a glance, J Cell Sci 115(Pt 20) (2002), 3861-3863.

[15] U. Koçak, U. Ezer and S. Vidinlisan, Serum fibronectin in neonatal sepsis: is it valuable in early diagnosis and outcome prediction? Acta Paediatr Jpn 39(4) (1997), 428-432.

[16] M.E. Lancer and G.E. Brown, Induction of hepatocytes synthesis of fibronectin by a non-interleukin 1 monokine, Journal of Trauma 28(8) (1988), 1220-1225.

[17] F. Boccardo, D. Guarneri, S. Zanardi, P. Castellani, L. Borsi and L. Zardi, Fibronectin concentration in the plasma of patients with malignant and benign breast disease, Cancer Lett 33(3) (1986), 317-323.

[18] S. Jung, G. Jung, A. Tranzer and R. Dörr, Blood fibronectin changes in various neoplasms, Presse Med 15(5) (1986), 197198, 203-204.

[19] C. Haglund, S. Ylätupa, P. Mertaniemi and P. Partanen, Cellular fibronectin concentration in the plasma of patients with malignant and benign diseases: a comparison with CA 19-9 and CEA, Br J Cancer 76(6) (1997), 777-783.

[20] J. Labat-Robert, Fibronectin in malignancy, Semin Cancer Biol 12(3) (2002), 187-195.

[21] F. Grinnell and M. Zhu, Identification of neutrophil elastase as the proteinase in burn wound fluid responsible for degradation of fibronectin, J Invest Dermatol 103(2) (1994), 155-161.

[22] M.L. Barilla and S.E. Carsons, Fibronectin fragments and their role in inflammatory arthritis, Semin Arthritis Rheum 29(4) (2000), 252-265.

[23] J.M. Guo, X.Y. Zhang, H.L. Chen, G.M. Wang and Y.K. Zhang, Structural alterations of sugar chains in urine fibronectin from bladder cancer patients and its enzymatic mechanism, J Cancer Res Clin Oncol 127(8) (2001), 512-519.

[24] A. Hegele, A. Heidenreich, J. Kropf, R. von Knobloch, Z. Varga, R. Hofmann and P. Olbert, Plasma levels of cellular fibronectin in patients with localized and metastatic renal cell carcinoma, Tumor Biol 25(3) (2004), 111-116.

[25] U.M. Warawdekar, S.M. Zingde, K.S. Iyer, P. Jagannath, A.R. Mehta and N.G. Mehta, Elevated levels and fragmented nature of cellular fibronectin in the plasma of gastrointestinal and head and neck cancer patients, Clin Chim Acta 372(1-2) (2006), 83-93.

[26] H. Sönmez, S. Süer, I. Karaarslan, H. Baloğlu and E. Kökoğlu, Tissue fibronectin levels of human prostatic cancer, as a tumor marker, Cancer Biochem Biophys 15(2) (1995), 107-110.

[27] M. Albrecht, H. Renneberg, G. Wennemuth, O. Möschler, M. Janssen, G. Aumüller and L. Konrad, Fibronectin in human prostatic cells in vivo and in vitro: expression, distribution, and pathological significance, Histochem Cell Biol 112(1) (1999), 51-61.

[28] S. Chakrabarty and H.A. Fritsche, Fibronectin and prostate cancer, Journal of Clinical Ligand Assay 25 (2002), 74-79.

[29] M. Morgan, S. Saba and W. Gower, Fibronectin influences cellular proliferation and apoptosis similarly in LNCaP and PC-3 prostate cancer cell lines, Urol Oncol 5(4) (2000), 155159.

[30] D.L. Livant, R.K. Brabec, K.J. Pienta, D.L. Allen, K. Kurachi, S. Markwart and A. Upadhyaya, Anti-invasive, antitumorigenic, and antimetastatic activities of the PHSCN sequence in prostate carcinoma, Cancer Res 60(2) (2000), 309-320.

[31] E. Ruoslahti, E.G. Hayman, M. Pierschbacher and E. Engvall, Fibronectin: purification, immunochemical properties, and 
biological activities, Methods Enzymol 82 (Pt A) (1982), 803831 .

[32] U. Laemmli, Cleavage of structural proteins during the assembly of the head of bacteriophage T4, Nature 227(1970), 680-685.

[33] P.C. Greenwood, M.W. Hunter and S. Glover, The preparation of ${ }^{131}$ I labelled growth hormone of high specific radioactivity, Biochem J 89 (1963), 114-120.

[34] Y. Gluzman, SV40-transformed simian cells support the replication of early SV40 mutants, Cell 23(1) (1981), 175-182.

[35] http://www.atcc.org/common/catalog/numSearch/ numResults.cfm?atccNum=CRL-1651.

[36] M. Hyytiäinen and J. Keski-Oja, Latent TGF-beta binding protein LTBP-2 decreases fibroblast adhesion to fibronectin, $J$ Cell Biol 163(6) (2003), 1363-1374.

[37] I. Katnik-Prastowska, M. Przybysz, A. Chełmońska-Soyta, Fibronectin fragments in human seminal plasma, Acta Biochim Pol 52(2) (2005), 557-560.

[38] M.M. Webber, A. Waghray and D. Bello, Prostate-specific antigen, a serine protease, facilitates human prostate cancer cell invasion, Clin Cancer Res 1(10) (1995), 1089-1094.

[39] http://www.copewithcytokines.de/cope.cgi?key=Fibronectin.

[40] Y. Yamaguchi, M. Isemura, M. Kosakai, A. Sato, M. Suzuki, M. Kan and Z. Yosizawa, Characterization of fibronectin from fetal human plasma in comparison with adult plasma fibronectin, Biochim Biophys Acta 790(1) (1984), 53-60.

[41] T. Krusius, M. Fukuda, A. Dell and E. Ruoslahti, Structure of the carbohydrate units of human amniotic fluid fibronectin, $J$ Biol Chem 260(7) (1985), 4110-4116.

[42] S. Carsons, B.B. Lavietes, A. Slomiany, H.S. Diamond and E. Berkowitz, Carbohydrate heterogeneity of fibronectins. Synovial fluid fibronectin resembles the form secreted by cultured synoviocytes but differs from the plasma form, $J$ Clin Invest 80(5) (1987), 1342-1349.

[43] E. Köttgen, B. Hell, C. Müller, F. Kainer and R. Tauber, Developmental changes in the glycosylation and binding properties of human fibronectins. Characterization of the glycan structures and ligand binding of human fibronectins from adult plasma, cord blood and amniotic fluid, Biol Chem Hoppe Seyler 370(12) (1989), 1285-1294.

[44] M. Takamoto, T. Endo, M. Isemura, N. Kochibe and A. Kobata, Structures of asparagine-linked oligosaccharides of human placental fibronectin, J Biochem (Tokyo) 105(5) (1989), 742-750.

[45] M. Takamoto, T. Endo, M. Isemura, Y. Yamaguchi, K. Okamura, N. Kochibe and A. Kobata, Detection of bisected biantennary form in the asparagine-linked oligosaccharides of fibronectin isolated from human term amniotic fluid, J Biochem (Tokyo) 106(2) (1989), 228-235.

[46] M. Tajiri, S. Yoshida and Y. Wada, Differential analysis of site-specific glycans on plasma and cellular fibronectins: application of a hydrophilic affinity method for glycopeptide enrichment, Glycobiology 15(12) (2005), 1332-1340.

[47] K. Murayama, E.J. Nichols, S.B. Levery, W.G. Carter and S. Hakomori, The carbohydrate structure of human fibronectins: A comparison between normal embryonic lung fibroblasts WI38 and the SV40 virus transformed cell line VA13, Glycoconjugate Journal 1(2) (1984).

[48] H. Matsuura and S. Hakomori, The oncofetal domain of fibronectin defined by monoclonal antibody FDC-6: its presence in fibronectins from fetal and tumor tissues and its absence in those from normal adult tissues and plasma, Proc Natl Acad Sci USA 82(19) (1985), 6517-6521.

[49] E.J. Nichols, B.A. Fenderson, W.G. Carter and S. Hakomori, Domain-specific distribution of carbohydrates in human fibronectins and the transformation-dependent translocation of branched type 2 chain defined by monoclonal antibody C6, $J$ Biol Chem 261(24) (1986), 11295-11301.

[50] H. Matsuura, T. Greene and S. Hakomori, An alpha-Nacetylgalactosaminylation at the threonine residue of a defined peptide sequence creates the oncofetal peptide epitope in human fibronectin, J Biol Chem 264(18) (1989), 10472-10476.

[51] U. Mandel, Hamilton, M. Therkildsen, J. Reibel, B. Sweeney, H. Matsuura, S. Hakomori, E. Dabelsteen and H. Clausen, Cancer-associated changes in glycosylation of fibronectin. Immunohistological localization of oncofetal fibronectin defined by monoclonal antibodies, APMIS 100(9) (1992), 817-826.

[52] http://home.comcast.net//kennethingham/newsite/intro.

[53] G.E. Jones, R.G. Arumugham and M.L Tanzer, Fibronectin glycosylation modulates fibroblast adhesion and spreading, $J$ Cell Biol 103(5) (1986), 1663-1670.

[54] A.J. Millis, M. Hoyle, D.M. Mann and M.J. Brennan, Incorporation of cellular and plasma fibronectins into smooth muscle cell extracellular matrix in vitro, Proc Natl Acad Sci USA 82(9) (1985), 2746-2750.

[55] F.A. Moretti, A.K. Chauhan, A. Iaconcig, F. Porro, F.E. Baralle and A.F. Muro, A major fraction of fibronectin present in the extracellular matrix of tissues is plasma-derived, $\mathrm{J}$ Biol Chem 282(38) (2007), 28057-28062.

[56] M. Yi and E. Ruoslahti, A fibronectin fragment inhibits tumor growth, angiogenesis, and metastasis, Proc Natl Acad Sci USA 98(2) (2001), 620-624.

[57] M. Fornaro, J. Plescia, S. Chheang, G. Tallini, Y.M. Zhu, M, King, D.C. Altieri and L.R. Languino, Fibronectin protects prostate cancer cells from tumor necrosis factor-alpha-induced apoptosis via the AKT/survivin pathway, J Biol Chem 278(50) (2003), 50402-50411.

[58] K.L. van Golen, L. Bao, G.J. Brewer, K.J. Pienta, J.M. Kamradt, D.L. Livant and S.D. Merajver, Suppression of tumor recurrence and metastasis by a combination of the PHSCN sequence and the antiangiogenic compound tetrathiomolybdate in prostate carcinoma, Neoplasia 4(5) (2002), 373-379. 


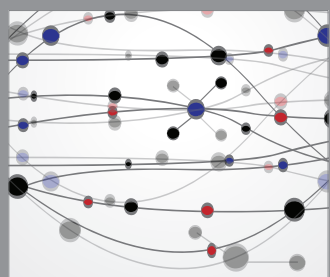

The Scientific World Journal
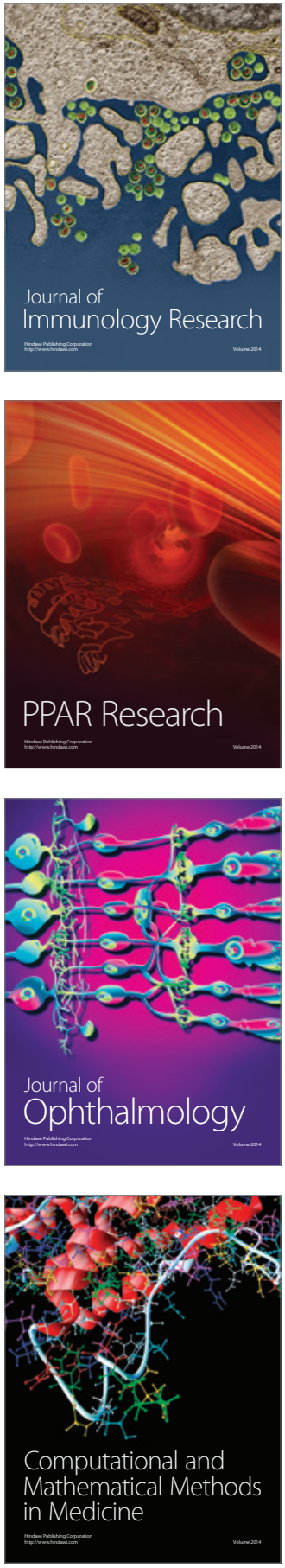

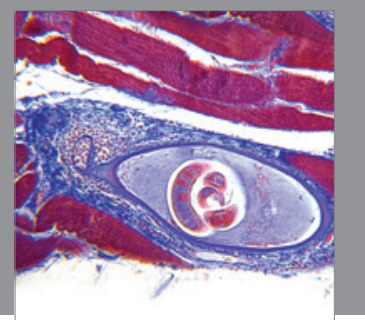

Gastroenterology

Research and Practice
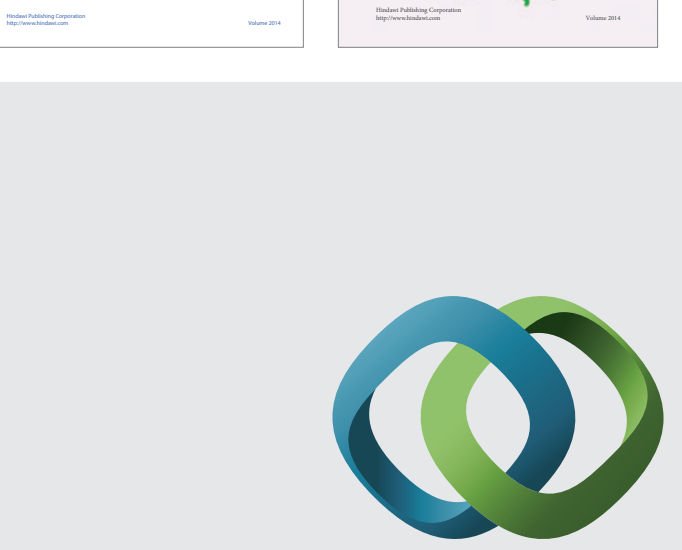

\section{Hindawi}

Submit your manuscripts at

http://www.hindawi.com
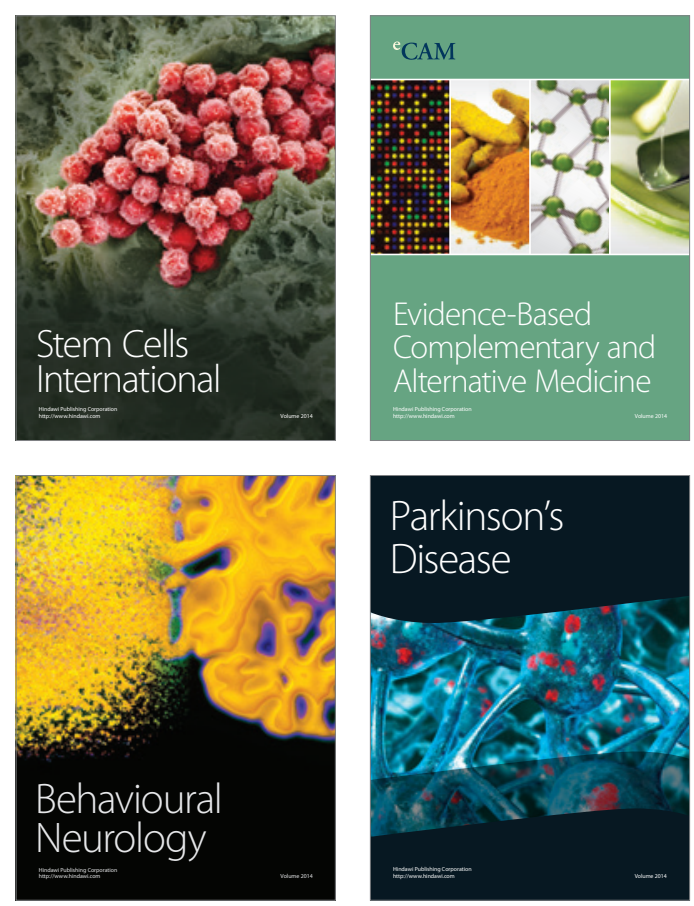

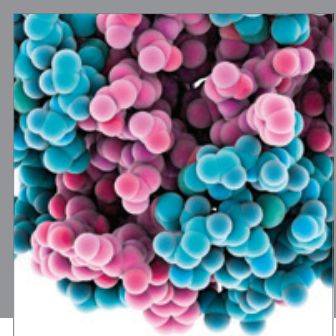

Journal of
Diabetes Research

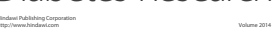

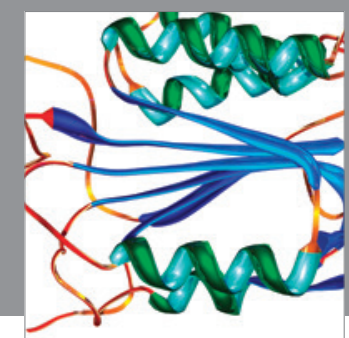

Disease Markers
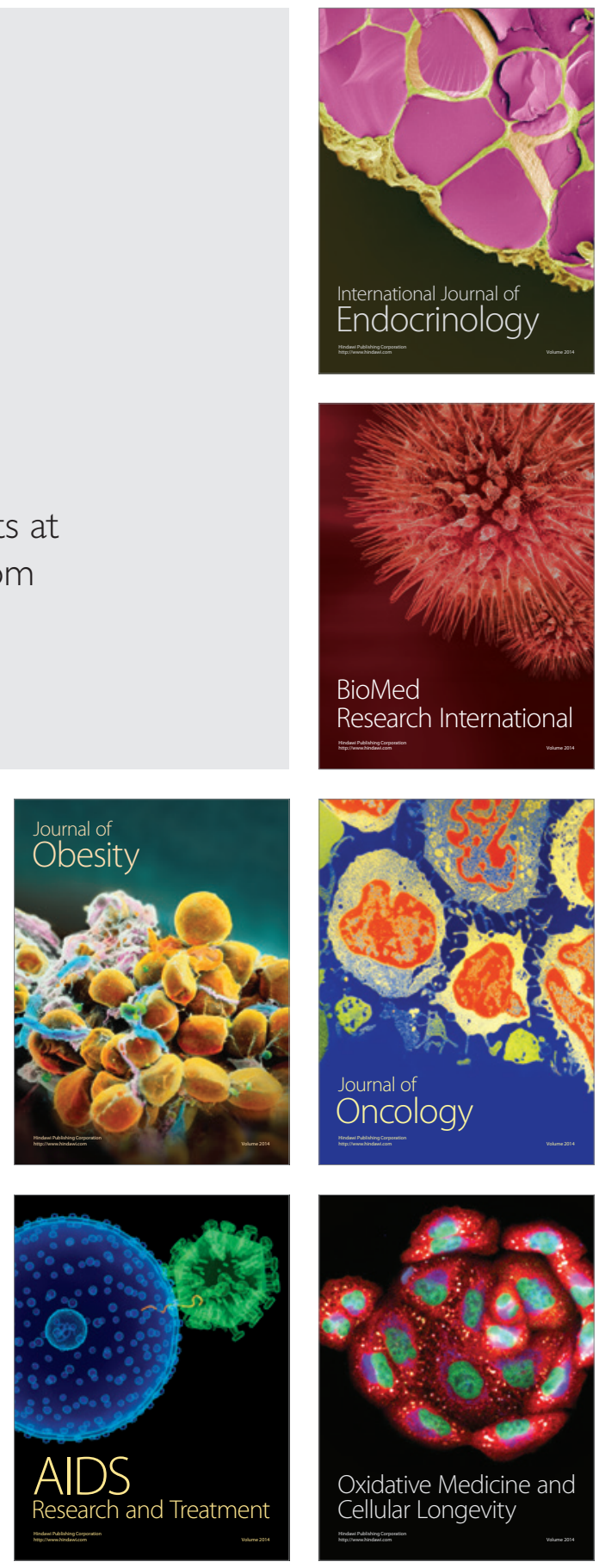